\title{
COHEN-MACAULAY RINGS AND CONSTRUCTIBLE POLYTOPES
}

\author{
BY RICHARD P. STANLEY 1
}

Communicated by Hyman Bass, July 15, 1974

We wish to point out how certain concepts in commutative algebra are of value in studying combinatorial properties of simplicial complexes. In particular, we obtain new restrictions on the $f$-vectors of simplicial convex polytopes.

Let $\Delta$ be a finite simplicial complex with vertex set $V=\left\{v_{1}, v_{2}\right.$, $\left.\cdots, v_{n}\right\}$. We call the elements of $\Delta$ the faces of $\Delta$. If the largest face of $\Delta$ has $d$ elements, then we say $\operatorname{dim} \Delta=d-1$. The $f$-vector of $\Delta$ is $\left(f_{0}, f_{1}, \cdots, f_{d-1}\right)$, where $\operatorname{dim} \Delta=d-1$ and exactly $f_{i}$ faces of $\Delta$ have $i+1$ elements. Define for positive integers $m$,

$$
H(\Delta, m)=\sum_{i=0}^{d-1} f_{i}\left(\begin{array}{c}
m-1 \\
i
\end{array}\right)
$$

Also define $H(\Delta, 0)=1$. We say that $\Delta$ is constructible [2] if it can be obtained by the following recursive procedure: (a) Every simplex is constructible, and (b) if $\Delta$ and $\Delta^{\prime}$ are constructible of the same dimension $d$, and if $\Delta \cap \Delta^{\prime}$ is constructible of dimension $d-1$, then $\Delta \cup \Delta^{\prime}$ is constructible.

We know of two main classes of constructible $\Delta$ 's: (A) The boundary complex of a simplicial convex polytope is constructible. This follows from [1]. (B) Let $D$ be a finite distributive lattice, and let $D^{\prime}$ be $D$ with the top element and bottom element removed. Let $\Delta$ be the simplicial complex whose faces are the chains of $D^{\prime}$. Then $\Delta$ is constructible.

If $h$ and $i$ are positive integers, then $h$ can be written uniquely in the form

AMS (MOS) subject classifications (1970). Primary 13C15, 52A25.

Key words and phrases. Simplicial complex, convex polytope, constructible polytope, upper bound conjecture, Hilbert function, Cohen-Macaulay ring, Gorenstein ring.

1 Supported in part by NSF Grant P36739. 


$$
h=\left(\begin{array}{c}
n_{i} \\
i
\end{array}\right)+\left(\begin{array}{c}
n_{i-1} \\
i-1
\end{array}\right)+\cdots+\left(\begin{array}{c}
n_{j} \\
j
\end{array}\right)
$$

where $n_{i}>n_{i-1}>\cdots>n_{j} \geqslant j \geqslant 1$. Following McMullen [5], define

$$
h^{\langle i\rangle}=\left(\begin{array}{c}
n_{i}+1 \\
i+1
\end{array}\right)+\left(\begin{array}{c}
n_{i-1}+1 \\
i
\end{array}\right)+\cdots+\left(\begin{array}{c}
n_{j}+1 \\
j+1
\end{array}\right) .
$$

Also define $0^{\langle i\rangle}=0$.

THEOREM 1. A vector $\left(f_{0}, f_{1}, \cdots, f_{d-1}\right)$ of positive integers is the f-vector of some constructible $\Delta$ of dimension $d-1$ if and only if $0 \leqslant h_{i+1} \leqslant h_{i}^{\langle i\rangle}, 1 \leqslant i \leqslant d-1$, where $h_{1}, h_{2}, \cdots, h_{d}$ are defined by

$$
\sum_{m=0}^{\infty} H(\Delta, m) x^{m}=\left(1+h_{1} x+h_{2} x^{2}+\cdots+h_{d} x^{d}\right) /(1-x)^{d}
$$

In the case where $\Delta$ is the boundary complex of a simplicial convex polytope, the numbers $h_{i}$ are equal to the numbers $g_{i-1}^{(d)}$ of McMullen [4]. Theorem 1 implies

$$
h_{i} \leqslant\left(\begin{array}{c}
f_{0}-d+i-1 \\
i
\end{array}\right)
$$

and is therefore a strengthening of the upper bound conjecture for convex polytopes (proved in [4]), and also a generalization to constructible polytopes.

We shall indicate the main idea used to prove the "only if" part of Theorem 1. Given $\Delta$ of dimension $d-1$, let $k$ be any field and let $R=k\left[v_{1}, v_{2}, \cdots, v_{n}\right]$ be the polynomial ring over $k$ whose variables are the vertices of $\Delta$. Define a homogeneous ideal $I$ of $R$ by taking for generators of $I$ all squarefree monomials $v_{i_{1}} v_{i_{2}} \cdots v_{i_{s}}$ with $\left\{v_{i_{1}}, v_{i_{2}}, \cdots\right.$, $\left.v_{i_{s}}\right\} \notin \Delta$. Let $A_{\Delta}=R / I$. It is easily seen that (Krull) $\operatorname{dim} A_{\Delta}=d$ and that $H(\Delta, m)$ is the Hilbert function of $A_{\Delta}$. By $\left[2\right.$, Theorem $\left.2^{\circ}\right], A_{\Delta}$ is Cohen-Macaulay (i.e., $h d_{R} A_{\Delta}=n-d$ ) if $\Delta$ is constructible. The "only if" part of Theorem 1 now follows from the following elaboration and generalization of a result of Macaulay [3].

THEOREM 2. Let $H(m)$ be a function from the nonnegative integers to the nonnegative integers. Let $0 \leqslant r \leqslant d \leqslant n$ be integers, and let $k$ be any field. The following two conditions are equivalent. 
(i) There is a homogeneous ideal I of $R=k\left[x_{1}, x_{2}, \cdots, x_{n}\right]$ such that if $A=R / I$, then $\operatorname{dim} A=d, h d_{R} A \leqslant n-r$, and $H(m)$ is the Hilbert function of $A$.

(ii) $H(0)=1 ; H(1) \leqslant n ; H(m)$ is a polynomial of degree $d-1$ for $m$ large; and $0 \leqslant h_{i+1, r} \leqslant h_{i, r}^{\langle i\rangle}, i \geqslant 1$, where

$$
(1-x)^{r} \sum_{m=0}^{\infty} H(m) x^{m}=\sum_{i=0}^{\infty} h_{i, r} x^{i}
$$

Conjecture 1. If $\Delta$ is as in (A) above, then $A_{\Delta}$ is Gorenstein.

Conjecture 2. Let $H(m), r=d, n$, and $k$ be as in Theorem 2. Let $h_{i}=h_{i, d}$ and $l_{i}=h_{i}-h_{i-1}, i \geqslant 1$. The following conditions are equivalent.

(i) There is a homogeneous ideal $I$ of $R=k\left[x_{1}, \cdots, x_{n}\right]$ such that if $A=R / I$, then $\operatorname{dim} A=d, A$ is Gorenstein, and $H(m)$ is the Hilbert function of $A$.

(ii) $H(0)=1 ; H(1) \leqslant n$; for some $t \geqslant 0, h_{t} \neq 0$ and $h_{s}=0$ if $s>t$; $h_{i}=h_{t-i}$ for $0 \leqslant i \leqslant t$; and $0 \leqslant l_{i+1} \leqslant l_{i}^{i\rangle}$ for $1 \leqslant i \leqslant[t / 2]$.

Conjectures 1 and 2 are closely related to the main conjecture of [5].

ADDED IN PROOF. Recent work of G. Reisner implies that $A_{\Delta}$ is Gorenstein when $|\Delta|$ is a sphere. This establishes Conjecture 1 and also implies the previously open "upper bound conjecture for spheres."

\section{REFERENCES}

1. H. Bruggesser and P. Mani, Shellable decompositions of cells and spheres, Math. Scand. 29 (1971), 197-205.

2. M. Hochster, Rings of invariants of tori, Cohen-Macaulay rings generated by monomials, and polytopes, Ann. of Math. (2) 96 (1972), 318-337. MR 46 \#3511.

3. F. S. Macaulay, Some properties of enumeration in the theory of modular systems, Proc. London Math. Soc. 26 (1927), $531-555$.

4. P. McMullen, The maximum numbers of faces of a convex polytope, Mathematika 17 (1970), 179-184. MR 44 \#921.

5. - The numbers of faces of simplicial polytopes, Israel J. Math. 9 (1971), 559-570. MR 43 \#3914.

DEPARTMENT OF MATHEMATICS, MASSACHUSETTS INSTITUTE OF TECHNOLOGY, CAMBRIDGE, MASSACHUSETTS 02139 